\title{
ESCRIBANOS Y NOTARIOS EN EL «REPARTIMENT» DE VALENCIA
}

\author{
M. ${ }^{\mathrm{a}}$ Desamparados Cabanes Pecourt \\ Universidad de Zaragoza
}

Este título tiene una doble significación al referirse, por una parte, a los escribanos y notarios que fueron favorecidos con algún dono en el reparto que de la ciudad de Valencia hiciera el rey conquistador, y por otra, a los autores materiales de los registros conocidos como «Repartiment del regne de Valencia». En esta ocasión trataremos tan sólo sobre el primero de estos aspectos, dejando el segundo para un futuro próximo.

Sobre quiénes fueron los escribas y notarios en los que recayeron los favores materiales de Jaime I en tierras de Valencia, la respuesta es variada ya que diversos son los personajes que con esta denominación puntual aparecen en los registros; y a ellos se pueden añadir algunos otros sospechosos de este ejercicio aunque no se explicite en la documentación.

La nómina de todos ellos es susceptible de diversas clasificaciones que en aras de una mayor claridad expositiva concretamos en los puntos siguientes:

A) escribanos y notarios reales.

B) escribanos y notarios de curia nobiliaria o eclesiástica.

C) escribanos y notarios profesionales.

A su vez, dentro del primer grupo se pueden distinguir los apartados siguientes:

1) notarios y escribanos, oficiales fijos de la cancillería real.

2) notarios y escribanos eventuales de la misma.

3) homónimos de notarios y escribanos reales de posible identidad con los mismos. 
De todos estos, de cuantos datos conozcamos sobre su biografía, haremos relación pormenorizada en un intento por conocer más y mejor a unos personajes que tanta repercusión tuvieron en su época y entre los que pueden encontrarse los autores materiales de las fuentes que hoy nos suministran los mayores puntos de conocimiento sobre la misma.

\section{A) ESCRIBANOS Y NOTARIOS DE LA CANCILLERIA REAL}

Incluimos entre ellos tanto a los que estaban al servicio del monarca como de cualquier otro miembro de la familia real. Distinguimos en primer lugar a los

1) escribanos y notarios reales especificados como tales en la documentación, puesto que permanecían de manera estable y fija en la cancilleria.

Presentan la siguiente nómina:

- PEDRO SANCHEZ, notario real.

- PEDRO JUAN, escribano real y notario de doña Violante.

- PEDRO ANDRES, escribano real.

- GUILLEM, escribano y notario real.

- GUILLEMON, escribano real.

- I. PEREZ, escribano ¿del infante Fernando?

- JUAN, "magister", notario de Nuño Sánchez.

- VALERO, «Scriptor Ferdinandi», «Scriba domini regis».

Hacemos a continuación un breve "curriculum" de cada uno de ellos, extraído de los datos que el "repartiment" proporciona, de los que conocemos por la documentación coetánea y de los escasos estudios que de la cancilleria del Conquistador hay hechos.

\section{Pedro Sánchez}

Aparece en el "repartiment" como "notarius domini regis". Ejerció este cargo de notario entre 1220 y 1232, simultaneándolo luego con el de repostero de Aragón entre esta última fecha y la de 1246 (1).

Son muchos los documentos reales que suscribe por lo que no vamos a citarlos aquí; basta con remitir a la colección de Documentos de Jaime I de Aragón (2) para confirmarlo.

Con motivo del reparto de la ciudad de Valencia, Pedro Sánchez fue favorecido en fecha muy temprana, pues del 28 de febrero de 1238 data la donación siguiente: «...totam hereditatem de Mahomat Algaschi et $X$ jovatas usque ad terminum de Petraher Açifilia vel hereditatem et domos d'Abrafim Abin luniç..." (3), donación que fue formalizada posteriormente ya que el asiento del "repartiment" está cancelado. 
Nuevamente lo encontramos en el registro correspondiente al censo de la ciudad, en el cual las citadas casas de Abrafim Abin luniç se ubican en el barrio de Teruel, diciendo así el asiento: «P. Sancii: domos Ebay Abeyunez et stabulum bonum et alfondec et mezquita et alia domo que querit ... IIII»(4), y si bien en este último no se menciona la profesión, la identidad entre las dos donaciones es evidente al venir reforzada por el nombre del sarraceno cuyas casas han sido entregadas al notario real, si bien la forma de ser anotado difiere un poco en su grafía al ser reseñado "de oido" por el correspondiente escriba real que hacía la anotación.

Reafirma este supuesto, asimismo, la $\mathrm{C}$ que, equivalente a Curia, precede marginalmente esta anotación y la segunda mención que de la misma donación se hace en la segunda parte del registro 7, en donde, al recogerse de nuevo la cita, dice: «P. Sanc, domini regis...» (5), con la misma $\mathrm{C}$ antecediendo al asiento.

Al margen de la heredad conque son agraciados en la ciudad y que es la revisada hasta ahora, los oficiales de la curia real se ven con gran frecuencia favorecidos con donos en el reino de Valencia, donde, generalmente, se les concede casas o tierras, o ambas, en las principales ciudades del reino que eran las que quedaban siempre en poder de la Corona. Pedro Sánchez obtuvo estos beneficios en poblaciones como Alcira, Cullera, Gandía y Beniopa (6), aunque desconocemos si las podría conservar, ya que, en el caso concreto de Alcira, se le da un plazo fijo para ir a poblar, perdiendo lo obtenido en caso contrario. Es de suponer que, al menos él, no vendría a vivir a tierras valencianas ya que, como al principio dijimos, permaneció al servicio del rey hasta 1246.

\section{Pedro Juan}

De Pedro Juan se sabe que fue escribano de la cancillería de Jaime I, cargo que ejerció desde 1224 hasta 1236 (7). De su mano salen numerosos documentos reales emitidos durante el sitio de Burriana, y desde esta misma ciudad ese mismo año y los siguientes (8); así como a él se debe la autoría material del tratado que en 1236 suscribieron el monarca aragonés y Zeit Abu Zeit (9). Actuó a las órdenes de los notarios G. Sasala y el anteriormente citado Pedro Sánchez.

En 1237 fue nombrado notario de la reina doña Violante (10) y con este título aparece ya en el «repartiment»: «notarius domine Yoles regine» cuando es favorecido con «domos d'Açmet Ambaraicha; et sunt domos in calle d'Almaxati, circa domos d'Albucach Alyfragi; et ortum in Villanova de alfaqui Avinomayhez, et IIII jovatas in alqueria de Petraher d'Alfauquia, iuxta hereditatem laquesii et G. Scribe" (11). Como en el caso anterior, esta donación tenía lugar antes de la conquista de Valencia y su entorno, pues está fechada el 16 de febrero de 1238, pero después de la conquista la donación se hizo efectiva, al menos en lo que respecta a las casas ubicadas en Valencia, estando éstas 
situadas en el barrio de Zaragoza. Dice asi el registro 7: «P. lohannis: domus Amet Ambarracar» (12), e igualmente recoge este nombre con posterioridad (13). En ambos casos es de señalar que aunque no se registra la profesión, si precede el asiento la $\mathrm{C}$ de curia como en el caso del notario anterior.

\section{Pedro Andrés}

Aparece como escriba de Jaime I desde 1247 a 1258 (14), desempeñando asimismo, en 1257, el cargo de notario de la ciudad de Huesca (15).

En documento del año 1251, datado en Lérida a 23 de julio, se intitula a sí mismo como «scriptoris domini regis et publici notarii» (16), y lo redacta por orden real directa.

Trabajó a las órdenes de los notarios G. Escriba, Guillem de Belloc, G. Pérez y del canciller Andrés, obispo de Valencia.

El citado Pedro Andrés, al igual que los dos anteriores, fue favorecido por el monarca aragonés en el reparto de las tierras y casas valencianas, recibiendo hornos, molinos y casas en lugares distintos a la capital del reino. En ésta se le entregan dos casas y se le denomina alternativamente «P. scriptor», "Petrus scriba» 0 «P. Andree». No es de extrañar que sólo en el reparto de Xátiva se le llame «scriptoris nostri», pues ello es debido a que el nombramiento real tiene lugar en 1247 y las donaciones de esta localidad ocurren en 1248, momento en que ya es escriba real.

Las casas recibidas en Valencia se encuentran situadas, una, en el barrio de Calatayud (17) y, la otra, en el de Zaragoza (18), y en ambos casos la $C$ precede al asiento así como se señala con una cruz, signo de que el beneficiario había tomado posesión de su dono.

\section{Guillem o Guillermo}

Puede datarse como escribano al servicio de Jaime I entre 1227 y 1247. Su actividad es intensa en la cancillería del conquistador y su signo aparece en la capitulación de la ciudad de Valencia (19). Ejerció su labor por mandato real en esta ciudad y en todo su reino y actuó bajo las órdenes de los notarios Guillem Sasala, G. de Almoguerra, Guillem Rabaça y Pedro Sánchez. En 1247 fue nombrado notario, cargo que ejerció hasta 1252.

Las donaciones que se mencionan en los registros reales concernientes al repartimiento de Valencia y relativas a este personaje son algo confusas por estar entremezcladas con las correspondientes a otro escriba real, Guillemón, pues son muchas las veces en que tan sólo se cita al escribano por la inicial de su nombre — la G-, que es común a ambos, y no faltan las ocasiones en que se menciona indistintamente al segundo como Guillém, Guillermo o Guillemón, lo que todavía complica más el asunto. 
No obstante, creemos que corresponde a este primer escribano unas casas sitas en el barrio de Zaragoza, casas de Ebray Alifraqui (20), que podian corresponderse con las entregadas a «G. scriba" en 28 de julio de 1237, aunque allí se denomine al sarraceno como Albucach Alyfragi (21), y en otra ocasión en que se alude al hecho de manera indirecta se le llame Abrafim (22).

Asimismo, parece corresponder a Guillem Scriba otra casa más, sita en el barrio de los hombres de Daroca, antigua posesión de Mahomat Almagledi (23), que quizá equivaliera a la dada a Salvador, en 1239, y que mediante rectificación es adjudicada a G. scriba (24).

\section{Guillemón}

Podemos documentarlo en la cancillería real ya en 1230 (25), aunque no lo hace de manera seguida hasta 1237 (26). Actuó bajo el mandato real del canciller Berenguer de Palou y de los notarios Guillermo de Belloc y Berenguer de Soler. Su última mención se contiene en documento de mayo de 1247 (27).

Según mención expresa del registro 7 (28), poseyó en la capital del reino seis casas: cuatro en el barrio de Teruel y dos en el de Barcelona.

Estas últimas -que unas veces aparecen a nombre de $G$. o de Guillém, y otras a las de Guillamó, organizando la confusión a la que aludiamos antes - fueron antes de Mahomat Alacir y quizá correspondan a las "casas de Alarif", sitas entre la puerta de la Xerea y de Bebaçachar, otorgadas en 1237 a nombre de Guillamón, scriba (30), donación que en ese momento fue anulada y reafirmada en tiempo posterior, el 2 de octubre del mismo año (31), expidiéndose en esta segunda ocasión el oportuno título de posesión.

Las del barrio de los hombres de Teruel, dos fueron antigua posesión de Mahomat Alguasqui y Alhag Abenhedar (32) (Mahomat Alguaschi y Alhaix Abenherder, según el registro 5) y se le concedieron a Guillemón el 10 de octubre de 1238 (33). También en este caso vemos aparecer junto al nombre de Guillemón el de Guillermo, y lo mismo ocurrirá con la reseña de las dos restantes casas, sitas asimismo en este barrio de Teruel, cuyos primitivos ocupantes fueron Mahomat Atelexi, Aturgelli y Açabaho (34), pero de las cuales desconocemos en qué momento pasaron a ser propiedad del escribano Guillemón.

Junto a estas donaciones de inmuebles, Guillemón se vio favorecido asimismo con la «scribaniam curie sive çalmedinati Valentie» el 7 de octubre de 1237 (35) y algunas otras tierras y posesiones en lugares próximos a la ciudad.

\section{Pérez}

Se le cita como «scriptor Ferdinandi» - del infante Fernando_, ocu- 
pando una casa en el barrio concedido a los hombres de Calatayud (36), aunque no pertenece a este origen.

No conocemos ningún dato sobre la identidad de este escriba ni tampoco lo encontramos asentado en el primer registro del "repartiment", donde teóricamente debiera hallarse la primigenia donación de las casas luego documentadas. En este registro aparece tan sólo un tal «l. Petri, Cesarauguste» (37) al que se le ofrecen unas casas y jovadas, pero del que no está nada claro que pueda tratarse de la misma persona.

En cuanto a la documentación real, tan rica en datos generalmente, sólo nos habla en un documento de Jaime I, de 1257, del nombramiento extendido a Juan Pérez, hijo de Pedro Sánchez de Fortón, como notario de Tarazona (38), el cual no creemos relacionado con el aquí tratado.

\section{Magister lohannes}

Es notario de Nuño Sánchez. Está documentado en el primer registro como beneficiario de casas, huerto y cuatro jovadas de tierra, donación a la que se añaden nuevas casas junto a estas últimas (39). Sin embargo, estas jovadas fueron posteriormente adjudicadas a Lope Ochoa, pues el citado "magistro" no vino en el plazo fijado a ocuparlas (40), lo que plantea seriamente la duda de que tampoco ocupara el resto de la donación.

El registro 7 no disipa tampoco totalmente esta duda, ya que si bien en él aparece un «maestro Juan» que, poseedor de unas casas en el barrio de Calatayud hace cesión de las mismas a B. de Spiels al no confirmar su residencia (41), no especifica que se trate del notario de Nuño Sánchez, lo que hacía en el primer caso, y además no hay coincidencia en los antiguos propietarios de las casas, por lo que casi desechamos que ambas fuentes estén refiriéndose a una misma persona.

\section{Valerio}

A la nómina antes expuesta podríamos añadir el presente escriba, Valerio, al cual se le denomina en ocasiones "scriptor Ferdinandi», y otras veces "scriba domini regis». Nada hemos podido saber de él, ni hemos obtenido ningún dato sobre su identidad. Podría tratarse de un escriba de Jaime I que en ocasiones sirviera también a su tío, el infante Fernando, pero también podría pertenecer al servicio de algún monarca castellano.

Ni entre los documentos del conquistador, en sus suscripciones cancillerescas, ni entre las nóminas de sus oficiales conocidos hemos podido localizarlo, así como tampoco en la documentación castellana.

Por otra parte, la existencia de un asiento (42) referido a «alium» que luego lleva tachada la frase "scriptore Ferdinandi" y luego sigue "lohannes, fratris Valerii» nos lleva a la conclusión de que se trate de un 
hermano del antes citado que, por su parentesco, se le cree con igual profesión y destino - «escriba de Fernando»-, cayendo luego en cuenta del error cometido. Esta confusión sirve al menos para confirmarnos la existencia real del primero, desechando la duda de que pudiera tratarse de una mala lectura.

En cuanto a los beneficios obtenidos por el tal Valerio, este escribano recibe el 31 de octubre de 1238 "domos de Abiafar de Arratoyl, iuxta domum de Alpico" (43), donación que está cancelada como signo evidente de la expedición documental.

Estas casas, al reflejarse en el censo de la ciudad siguen siendo contiguas a la "domus alchait Alpic» (44), pero, en cambio, no coinciden con el nombre del antiguo propietario, pues ahora se atribuyen a Hamet Amnatavel (45). Las casas están ubicadas en el barrio de Lérida.

Pero pese a todo lo dicho, parece ser que Valerio no permaneció en Valencia, pues dice el texto que «...non habet hereditatem et non in presentibus negue in absentibus... et habet ante cartam episcopi Barchinone" (46), y de nuevo insiste más tarde: "...et aparuit cartam episcopi Barchinone... //» (47).

Posible justificación a esta ausencia podemos encontrarla en el registro 6, registro que computa las donaciones hechas en diversos lugares del reino, en donde "Valerio, scriptori", junto con otros individuos recibe casas en Xátiva y tierras en Benimantell y Beniabdon (48) y nuevas tierras en la primera (49), donde quizá se asentara.

$Y$ hasta aquí los oficiales que hacen constar expresamente su adhesión a la cancillería regia como titulares de la misma, pasando, a continuación, a considerar aquellos que sólo intervenían de manera ocasional en la confección de los diplomas reales.

2) ESCRIBANOS Y NOTARIOS EVENTUALES DE LA CANCILLERIA REAL

Son, por orden alfabético:

- GUILLERMO GALCERAN.

- GUILLERMO VIDAL.

- PONCIO CARBONELL.

\section{Guillermo Galcerán}

Notario de Valencia y autor ocasional de los documentos de Jaime I, no recibió su nombramiento como tal hasta el año 1240 (50). Su actividad fue enorme en la ciudad en la década subsiguiente a la conquista (51), y en 1242 lo vemos suscribir en un diploma real representando a un canónigo de la sede valenciana (52); pero dado que cuando la ciudad fue 
censada el tal Guillermo Galcerán aún no habia conseguido su "status" de notario, cualquiera de los homónimos que aparezcan en el «repartiment" puede referirse a este mismo personaje.

Las citas que aparecen en esta fuente referidas a individuos con este nombre son las siguientes: heredado en la ciudad de Valencia, y censado en ella, con fecha anterior a mayo de 1240 se nombra a un Guillém Galcerán que recibió la casa de Atec Abisalamo, sita en el barrio de Zaragoza (53); no consta, sin embargo, la fecha real del otorgamiento.

Con fecha 18 de mayo de este mismo año 1240 hay una concesión al "magister $G$. de Gaucera" de las casas que, sitas en la parroquia de santa María de Barcelona, habian sido entregadas con anterioridad a A. de Alfo, el cual no se había presentado para su ocupación en el término establecido, por lo que le es revocada la entrega (54). La casa linda con la de Guirad Roger, el cual tampoco vino, al parecer, ya que el texto dice «...uixta domos que fuerunt G. Rogerii...». El hecho de que el censo de la ciudad recogido en el registro 7 sea hecho con anterioridad a la fecha del 18 de mayo de 1240 , fecha de la donación a G. de Gaucera, es la causa de que en aquél todavía aparezcan como detentadores de las mencionadas casas Arnau de Alfo (55) y Guirad Roger (56).

Por último, está el único testimonio de entrega a un notario de nombre G. Gaucerá. También es posterior a la redacción del censo contenido en el registro 7 y está expresada en dos asientos: el uno anulado, fechado el 17 de agosto de 1240 y expedido a nombre de G. Gaucelmi. El segundo, un día más tarde, válido y dirigido a "G. Gaucera, notarius» (57).

Posiblemente este personaje fuera el mismo que el sujeto de la anterior donación, el maestro $\mathrm{G}$. de Gaucera.

\section{Guillermo Vidal}

Parece que es también un escriba ocasional de Jaime 1. El «repartiment» Io cita como "Vitalis, scriptor dertusensis" $y$ "G. Vidal, scriptor»; no obstante, la documentación de Jaime l lo intitula «notarius publicus dertusensis» y se le ve aparecer casi coetáneamente al recibo de los donos reales redactando el testamento de doña Toda Ladrón, esposa de Gil Garcés, por orden del conquistador que a su vez lo suscribe (58). Algunos años antes, en 1234, había validado la carta puebla del castillo de Xivert, concedida por el maestre del Temple (59), y algunos años más tarde, redacta el documento de vasallaje que algunos ricos-hombres aragoneses debian prestar al monarca (60).

Las casas recibidas en la ciudad de Valencia lo son en el barrio de Barcelona, y pertenecian a Ali Alamello. De esta donación se consignan varios asientos anulados (61) y un último válido (62), datado en febrero de 1238.

Una vez redactado el censo de casas, el nombre de «Vidal» se da como 
ocupante de este mismo inmueble, precedido el asiento de la $\mathrm{C}$ preceptiva y la señal de haber hecho una ocupación real de la misma (63). Sin embargo, parece ser que con posterioridad la abandonó y fue entregada a Ferrer de Caraut (64).

¿Cuál pudo ser el motivo del abandono? Una posible justificación sería el nombramiento o concesión que Jaime I hizo del justiciadgo de Sagunto a una tal G. Vidal (65), caso de tratarse de la misma persona, pero esto tan sólo son conjeturas y meras hipótesis.

\section{Poncio Carbonell}

De acuerdo con la bibliografía publicada (66), existe en 1246 un escriba ocasional del monarca aragonés denominado Poncio Carbonell, el cual es notario de Valencia.

Entre la nómina que ofrece el «repartiment" de los ejercientes de dicho oficio, no consta ninguno de este nombre entre los heredados en Valencia ciudad, pero si en la ciudad de Xátiva. En asiento datado el 13 de abril de 1248 aparece un tal "Poncius Carbonelli, scriptor", residiendo en dicha ciudad (67); y, asimismo, diez años más tarde, es notario público de Xátiva un individuo portador del mismo apellido, “Petrus Carbonelli» (68).

Desconocemos si el Poncio Carbonell, escriba ocasional, y el asentado en Xátiva pudo ser el mismo, pero lo que sí es claro es que este segundo se encuadra dentro del tema que nos ocupa, aunque su sitio debiera ser más correcto trasladado al último punto del trabajo.

3) Homónimos de escribanos y notarios reales. Homónimos de estos oficiales aparecen en el "repartiment", pero sin expresión de su cargo, por lo que no puede asegurarse su identidad, no obstante los ofrecemos aquí. Son los siguientes:

- BERNARDO DE SOLER.

- GUILLERMO DE BELLOCH.

- GUILLERMO DE OLESA.

\section{Bernardo de Soler}

Recibe una casa en el barrio de Lérida (69), entregada el 13 de junio de $1238(70)$, y es llamado por la documentación «magister» aunque no notario. Su posible credencial como componente de la casa real es la $\mathrm{C}$ que precede al asiento de su nombre.

Esta misma donación es repetida en diciembre de 1239 (71), aunque fue anulada; suponemos que por estar ya expedida el acta con anterioridad. 
Este Bernardo de Soler actuó como notario real entre 1243 y 1247 , siendo notario mayor en este último año citado (72).

La colección diplomática de Jaime I nos lo muestra suscribiendo dos documentos datados en Huesca, en 21 y 28 de julio de 1247, relacionados con la orden del Temple, a la que posiblemente perteneciera este «magister» (73).

\section{Guillermo de Belloch}

Está documentado como notario real entre 1241-1251, ejerciendo de notario mayor desde la primera fecha hasta 1246. Tuvo a sus órdenes a los escribanos Pedro Andrés, Guillemó y Guillermo Sarroca.

En el «repartiment» aparece como perteneciente a la curia real, aunque sin título de notario, cosa lógica puesto que este nombramiento se le concede en 1241 y la fecha de entrega de dos casas en Valencia, en el barrio de Lérida, una grande y otra pequeña, es de 10 de julio de 1238 (75). La donación se hace a nombre de G. de Pulcroloco.

\section{Guillermo de Olesa}

Notario público de Barcelona y escribano ocasional en la cancillería real, está documentado entre 1225 y 1226, y creemos más que probable que fuera el beneficiado con la casa que había sido de Ali Acilevi, sita en el barrio que se dio a los habitantes de Barcelona. De este Guillém de Olesa se hace constar su origen barcelonés (76), pero no conocemos el momento de la donación.

\section{B) ESCRIBANOS Y NOTARIOS DE CURIA NOBILIARIA O ECLESIASTICA}

Este segundo grupo lo constituyen una serie de escribanos al servicio de la nobleza aragonesa y de la Iglesia. Los datos que sobre los mismos poseemos son menores, obviamente, que los del grupo anterior.

Son escribanos de este tipo:

- GARCIA, escribano de Rodrigo de Lizana.

- MARTIN PEREZ, escribano de Jimeno Pérez de Arenós.

- P. DE CUBELLS, escribano del Temple.

- P. MOREILO, escribano de Artal de Alagón.

- GARCIA, escribano de Rodrigo de Lizana.

De él tan sólo conocemos que obtuvo, el 7 de mayo de 1238, casas en Valencia, más cuatro jovadas en el término de esta ciudad, y huerto (77), donación que aparece cancelada como de haber sido hecha efectiva, lo que queda corroborado con la mención de unas casas de su pertenencia 
en el barrio de Lérida (78), antigua posesión de Ebrahim Ebnorella, alatror. Sin embargo, este escriba no permaneció en este lugar, puesto que la documentación dice que "non erat hic nec ostendit carta», ampliando la información al decir que pasó a manos del obispo de Tortosa: «dedit eam episcopo dertusensi» (79).

\section{Martínez Pérez, escriba}

El documento que consigna el pleito-homenaje que Gil de Rada hiciera a Jaime I por la tenencia del castillo de Rada está suscrito por un escriba de nombre Marti Petri, quien manifiesta escribir este diploma por "mandato domini regis, dompni Egidii de Rada et dompne Marie de Leet» (80). Un tiempo más tarde, el 23 de enero de 1258, en la suscripción de otro diploma del mismo monarca, podemos leer: «...per manum Martin Petri, scribe Eximini Petri de Arenoso et notarii publici Valencie...» (81).

Es decir, existió un Martín Pérez que fue notario público de Valencia, escribano de Jimeno Pérez de Arenós y escribano ocasional de Jaime I. Todas estas ocupaciones eran fáciles de asumir sin perjuicio para ninguna de ellas, ya que este noble aragonés se afincó en el reino de Valencia, donde tenia un amplio señorio —el de Arenós- en provincia de Castellón, y sus hijas profesaron en el monasterio de Santa Clara de la ciudad de Valencia, con el cual estuvo muy vinculado.

Ahora bien, hallar a este Martín Pérez entre los habitantes censados en Valencia resulta tarea algo ardua, ya que no aparece ningún personaje con este nombre que detrás lleve el apelativo de escriba.

Con este nombre aparecen en el «repartiment» diversos propietarios de casas.

En el barrio de Calatayud hay dos menciones, una de las cuales incluye la posesión de dos casas (82); en el barrio de Zaragoza dos casas más, pero bajo un único asiento (83); en el barrio de Daroca otra más (84). Desconocemos si alguna de todas estas citas, o cuál de estas citas puede corresponder a nuestro personaje, aunque es de suponer que alguna de ellas le alude.

\section{P. de Cube//s}

Es un escriba de la orden del Temple. Los donos con que se vio favorecido P. de Cubells en el reparto de Valencia se inician en 1237 (85), si bien esta primera donación, aunque lleva la indicación de haberse expedido el correspondiente documento justificativo, aparece anulada por una serie de barras, y será algún tiempo más tarde, el 28 de octubre de 1238, cuando de nuevo recibirá las "domos de Mahomat Avincoto ... et II iovatas in Autel et III fanecatas terre pro orto in Ramb/a..." (86). Estas casas - son dos- constan en el registro 7 ubicadas en el barrio de Lérida (87). 
Fue favorecido con «domos Mahomat Alcuilari et Loba uxore sue», a las que se añadió un huerto y tres jovadas, donación que tuvo lugar el 2 de mayo de 1238 (88). El asiento está cancelado como muestra de que la donación fue hecha efectiva; sin embargo, en la relación de casas de Valencia no la hallamos. Alli aparece un tal P. Moriello (89), pero las casas que posee señalan como antiguo dueño a Ebrahi Ebinhalela Alquietni y no al antes citado, por tanto queda planteada una duda razonable de que no se trate de la misma persona.

\section{C) ESCRIBANOS Y NOTARIO PROFESIONALES}

Por último, nada más queda por reseñar a los profesionales, de los cuales podemos aportar poco más que su nombre, origen en su caso, y dono que les correspondió.

Son los siguientes:

- B., escriba de Burriana.

- BARTOLOME, escriba de Mora.

- BERNARDO, escriba.

- BERTRANDO, scriptor.

- I. o JUAN, escriba.

- I. MARTIZ, escriba, navarro.

- IOANOT DE VERBEgal.

- MARIA PETRI, escriba.

- MARTIN DE MONTALBAN, scriba.

- P. FERRER, notario de Barcelona.

- PONCIO DE ClaRAVAL, escribano de Lérida.

- R. BONIFACI, escribano.

\section{B., escribano de Burriana}

No obtuvo casa en la capital del reino, sino tan sólo un molino de cuatro ruedas contiguo a una viña (90), según donación fechada el 27 de octubre de 1240. Se canceló por expedición del documento.

Bernardo, escribano

Aunque existe un "Bernardo, scriptorem» a quien la reina doña Violante, esposa de Jaime I, recuerda en su testamento, asignándole un dinero a recibir sobre la bailía de Prades (91), en 1251, y suponemos que existirian muchos más a lo largo y ancho del territorio de la corona aragonesa, nos inclinamos en el presente caso a creer que no se trata de un escriba, sino de un personaje apellidado así. 
Parece ser que este Bernardo Escribá aquí mencionado pertenecía a la curia real, a juzgar por la $\mathrm{C}$ indicada al margen, y recibió casas en el barrio sito entre Daroca y Teruel (92), «iuxta portam de Alcantera» (93), casas que le fueron entregadas el 5 de mayo de 1239.

Pudo tratarse con mayor seguridad del rebostero real del mismo nombre, por lo que no debe contabilizarse entre los profesionales aquí estudiados.

\section{Bertrando, escribano}

Igualmente desconocemos cualquier dato o circunstancia que se refiera al denominado "Betrandus, scriptor", el cual recibe, el 19 de octubre de 1238, la casa de Abdela Alcaray, tejedor (94); pues esta misma casa, al reflejarse en el censo pormenorizado del registro 7, aparece situada en el barrio de Zaragoza, viene precedida de una $C$, pero su nuevo poseedor, aunque también llamado Bertrán, se apellida «de Balager» (95), por lo que no hay seguridad de que se trate del mismo personaje.

Caso de que este Bertrandus se asentara en Valencia efectivamente de forma definitiva, podría ser el que, algunos años más tarde, actuara como notario de esta capital (96), o bien se tratara de Bernardo de Balaguer, igualmente notario público de Valencia, que ejerció su oficio en los años cuarenta (97).

\section{I., escribano}

No sabemos quién pudo ser este "scriptor". Puede sospecharse que perteneciera a la cancilleria real porque en la casa que se le concedió en Valencia, en el barrio de Calatayud (98), hay una $R$ precediendo el asiento, lo que hace suponer que es uno de los inmuebles reservados por el rey y que luego iba entregando a personajes muy relacionados con él mismo; o a los que debía algún favor. No obstante, caso de ser cierto este supuesto, no sabemos a quién podria corresponder la identidad de este escriba real, ¿a Jaime de Montjuich?, ¿a Jaime de Rupe? Lo único seguro es que «l., scriptor» recibió esta casa junto con tierras, en el arrabal Axuterni, el 22 de abril de 1238 .

\section{Martiz, escribano, navarro}

Se contabiliza junto a otros cinco individuos en una donación fechada el 1 de junio de 1239, por la que se les entregan las casas de la villa de Roteros "ad populandum", para que ellos se encarguen de "dividendum navarris qui ibi populaverint» (100).

La donación está cancelada como signo de haber sido hecha efectiva; sin embargo, nos encontramos con que no hay confirmación de la estancia de este personaje en el registro 7, ya que éste acabó de redactarse en mayo del mismo año, o sea, con anterioridad. 
Aparece censado como propietario de una casa en Valencia, antigua posesión de Ali Abençada (101), desconociéndose cualquier circunstancia sobre su concesión.

El inmueble estaba dentro de los límites del barrio concedido a los hombres de Daroca y éste parece que era el origen del mencionado notario.

Maria Petri, escriba

En el presente caso, más que un escribano, creemos que esta señora se apellidaba así, pero se da la circunstancia de que por dos veces se la cita de esta manera (102) y una tercera como "MARIA Scribani" (103), por lo que no queremos dejar de incluiria aquí. Las casas las poseía en el barrio de la Carnicería.

Martín de Montalbán, escribano

Procedente de Teruel, recibe, en 1238, casas en Valencia y tierras en Campanar (104). Las casas son dos, pertenecen a Mahomat Alcaraquivi y Mahomat Amanazili, y están situadas en barrio de Teruel. Pero el dicho Martín de Montalbán no se quedó en la ciudad y su dono pasó a manos de Benedicta (105).

\section{P. Ferrer, notario de Barcelona}

Le son concedidas "domos de Mahomat Exarahabi et ortum" en agosto de 1238 (106), según asiento cancelado y tachado con posterioridad. Dichas casas no coinciden con ninguna de las censadas en el registro 7 en poder de homónimos suyos - y mucho menos con ninguno de quien se cita el oficio de notario-, lo que confirma la anulación expresada con la tachadura y justifica la ausencia.

\section{Poncio de Claraval, escribano de Lérida}

El 18 de mayo de 1238 obtiene casas en Valencia y tierras en Ruzafa, lo que se anota en un asiento sin cancelar (107). Tampoco se halla censado, por lo que es de suponer que no se le haría efectiva la donación.

\section{R. Bonifaci, escribano}

Debe tratarse del que, con el nombre de Bonifacio, aparece mencionado en el registro 5 y al que se le conceden adomos in vico de Albanec et fuerunt de Açiça, filia de Sança, de Amet Alcauz et de Mahomat Ambona, et II iovatas ... et contiguantur cum honore P. Maçip et Berengarii de 
Monteregali et in honore Berengarii Mercer de Vallibus ... cum reallo episcopi Barchinone...» (108), y al que en el registro 7 ya se le menciona el oficio, al enumerar las casas en el barrio de la Carniceria, donde especifica: «Bonifaci, scriptor, domos Haynona» (109). Suponemos que la diferencia apreciada en el nombre del sarraceno se debe a la percepción del escriba y que se corresponderán las casas con las del anteriormente citado Ambona. En la repetición del asiento ya se le cita como «R. Bonifaci» (110).

Finalizada ya la enumeración de los notarios y escribanos a quien el rey gratificara con donos en el reparto de Valencia podemos concluir con el siguiente balance:

- El total de personas beneficiadas con este trato alcanzan casi la treintena, de las cuales las más favorecidas con: Guillemón con seis casas, seguido de García Sánchez con cuatro y Guillém y el notario de Nuño Sánchez con tres. El resto reciben dos y un inmueble.

- Todos estos inmuebles están ubicados, el mayor número en el barrio de los hombres de Teruel, que contabiliza 10 casas más una en el barrio intermedio entre Teruel y Daroca, seguido del de Zaragoza con nueve y Calatayud y Lérida con siete. El menor número lo computa el barrio de los hombres de Tortosa, con un inmueble, y el de las Carnicerías, con dos. El de Daroca y Barcelona cuentan con tres y cinco casas entregadas, respectivamente.

Por último, la permanencia de los agraciados en la capital valenciana es una afirmación muy delicada de hacer. Parece claro que no permanecieron el Maestro Juan, notario de Nuño Sánchez; Valerio, Guillermo Vidal, Poncio Carbonell, Martín de Montalbán, García, el escribano de Burriana, P. Ferrer, el notario de Barcelona, Poncio de Claraval y, posiblemente, I. Martiz; unos porque no vieron confirmada su donación; otros porque - debido a diferentes causas - traspasaron su dono a un nuevo propietario.

Es de suponer que tampoco se asentarian aquellos oficiales que continuaron en la cancillería regia y como tales acompañaban al rey, como es el caso de Pedro Sánchez, Guillém y Guillemón, Bernardo de Soler y Guillermo de Belloch.

Se establecerían posiblemente I. Pérez, Guillermo de Olesa, R. Bonifacio y loanot de Vérbegal, si atendemos a los signos que acompañando a los respectivos asientos así lo indican, más Guillermo Galcerán y Martín Pérez, que, como ya quedó dicho, ejercieron su actividad notarial en Valencia. Por lo que respecta a Pedro Andrés, como su servicio al rey se inicia en 1247, pudo residir en Valencia desde el momento de la conquista hasta dicha fecha, en el supuesto de que ambos personajes, el del "repartiment" y el oficial real, sean la misma persona. 


\section{NOTAS}

(1) Cfr. TRENCHS ODENA, J., La cancilleria de Jaime I: cancilleres y escribanos, en «Studi in onore di Giulio Battelliw. Roma, 1979, pags. 117 y 121.

(2) Cfr. HUICI, A., y CABANES, M.* D., Documentos de Jaime l de Aragón, I, II. III.

(3) Cfr. CABANES, M.* D., y FERRER NAVARRO. R., Libre del Repartiment del Regne de Valencia, Zaragoza, 1979, I, asiento 145 (en adelante, 1-145).

(4) Cfr. CABANES Y FERRER, Repartiment, III-2.265, 3346.

(5) Ibid., III-3346.

(6) Ibid., It-961, 1.209, 1.097 y 1.328.

(7) Cfr. HUICI Y CABANES. Documentos de Jaime I. I.

(8) Ib/d., I, núms. 182 a 185, 189, 190, 201...

(9) Ibid., I, núm. 236.

(10) Ibio.. I, núm. 224.

(11) Ctr. CABANES Y FERRER, Repartiment. -338.

(12) Ibid., IH-855.

(13) Ibid., III-2.749.

(14) Cfr. HUICI Y CABANES. Documentos de Jaime I, II, núm. 471. IV, núm. 984.

(15) Cfr. TRENCHS, La cencilleria, pag. 119.

(16) Cfr. HUICI Y CABANES, Documentos de Jaime I, III, núm. 575.

(17) Ctr. CABANES Y FERRER, Repartiment. 1-846, IIt-1.457, 2.977.

(18) Ibid., 1-837, III-854, 2.748.

(19) Ctr. HUICI Y CABANES, Documentos de Jaime I, I, nüm

(20) Ctr. CABANES Y FERRER, Repartiment, lii-853, 2.747.

(21) Ibid., 1-27.

(22) Ibid., 1-990.

(23) Ibid., IIt-2.111 y 2.832

(24) Ibid., 1-1.258.

(25) Cfr. HUICI Y CABANES, Documentos de Jalme I, I. num. 143.

(26) Ibid., I, núm. 245 y sigs

(27) Ibid. I, núm. 459.

(28) Cfr. CABANES Y FERRER. Repartiment, III-3.347.

(29) Ib/d., III-5 y 2.495.

(30) Ibid., 184.

(31) lbid., $1-87$.

(32) Ibid., III-2.266 y 3.347 .

(33) Ibid., 1014 .

(34) 1bid., III-2.297.

(35) Ibid., 183.

(36) Ibid., III- 1.490 y 2.998.

(37) Ibld. $1-962$.

(38) Ctr. HUICI Y CABANES, Documentos de Jaime 1, III-775.

(39) Cfr. CABANES Y FERRER, Repartment. +420 . 
(40) Ibid., 1-591

(41) lbid., lil-1.497 y 3.003

(42) Ibio., III-1.491.

(43) loid., 1.017.

(44) Ibid., Ill-1.796.

(45) Ibid., III-1.797.

(46) Ioid., III-1.797.

(47) Ibid., III-3.162.

(48) Ibid., II-703 y 1.209.

(49) Ibio.. It-849.

(50) Cfr. HUICI Y CABANES. Documentos de Jaime I. II. núms. 352. 353.

(51) Cfr. AHN. Clero. Carp. 3.271 nums. 13 y 15; carp 3.272, nums. 7. 10, 11, 16 y 16 , carp. 3.273. num 8: carp. 3.274, nums. 12, 18, etc

(52) Cfr. HUICl Y CABANES. Documentos de Jaime I, H, nums 352.353

(53) Cfr. CABANES y FERRER, Repartiment, III-860

(54) Ibid., 1-1.549.

(55) Ibid., III-114.

(56) Ib/d., III-115.

(57) Ibid., l-1.666.

(58) Cfr. HUICI y CABANES, Documentos de Jaime I, II, 272.

(59) Cfr. AHN. O. Montesa, cod. 542, fol. 26 v.

(60) Cfr. HUICl y CABANES, Documentos de Jaime I, II, 315.

(61) Cfr. CABANES y FERRER, Repartiment, l-138 y 151.

(62) Ibid., I-152.

(63) Ibid., III-90.

(64) Ibid., III-2.570.

(65) Ib/d., Il-439.

(66) Cfr. TRENCHS, La cancilleria, pág. 119.

(67) Cfr. CABANES y FERRER, Repartiment, II-638.

(68) Cfr. AHN. Clero, carp. 3.274, núm. 9.

(69) Cfr. CABANES y FERRER, Repartiment, III-1.743.

(70) Ibid., l-363.

(71) Ibid., I-1.516.

(72) Cfr. TRENCHS, La cancillerla, págs. 117 y 121.

(73) Cfr. HUICI y CABANES, Documentos de Jaime I, III-466, 467.

(74) Ibid., I-IV.

(75) Cfr. CABANES y FERRER, Repartiment, I-467; III-1.677 y 349.

(76) Ibid., III-188.

(77) Cfr. Ibid., I-281.

(78) Ibid., IIt-1.885.

(79) Ibid., III-3.173.

(80) Cfr. HUICl y CABANES, Documentos de Jaime I, III-862.

(81) Ibid., IV, 942.

(82) Cfr. CABANES y FERRER, Repartiment, Ili-1.440 y 1.447.

(83) Ibid., III-775 y 2.720.

(84) Ib/d., III-1.986.

(85) Ibid., I-62.

(86) Ibid., 1-1.005.

(87) Ibid., III-1.877.

(88) Ibid., I-247.

(89) Ibid., Ill-1.055.

(90) Ibid., I-127.

(91) Cfr. HUICI y CABANES, Documentos de Jaime I, Ill-585.

(92) CABANES y FERRER, Repartiment, III-2.149 y 3.293.

(93) Ibid., I-1.445.

(94) Ibid., 1-941.

(95) Ibid., III-709.

(96) Cfr. ARV.

(97) Cfr. AHN. Carp. 3.271, núms. 14 y 17.

(98) Cfr. CABANES y FËRRER, Repartiment, III-1.229. 
(99) Ibid., 1-208.

(100) lb/d., l-1.304.

(101) Ibid., III-1.997.

(102) Ib/d., IHI-1.112.

(103) Ibid., l-1.615.

(104) Ibid., I-141.

(105) Ibid., III-2.379.

(106) Ibid., 1-580.

(107) Ibid., I-291.

(108) Ibid., l-1.395.

(109) Ibid., III-1.102.

(110) Ibld., III-2.823. 